\title{
WRC: Production technology drives Motorsport, Formula 1: Motorsport drives production technology
}

Donatus Wichelhaus, Volkswagen Motorsport GmbH

This manuscript is not available according to publishing restriction. Thank you for your understanding. 